\title{
Pulmonary nocardiosis caused by Nocardia otitidiscaviarum in an adult asthmatic female patient: The presence of acid-fast branching filaments is always significant
}

\author{
A Mahgoub, ${ }^{1}$ DMLT, BSc; S A Gumaa, ${ }^{2} \mathrm{MD}$, PhD; M R P Joseph, ${ }^{3} \mathrm{BSc}, \mathrm{MSc}, \mathrm{PhD} ; \mathbf{M}$ S Saleh, ${ }^{4} \mathrm{BSc}, \mathrm{MSc}$; A H A Elsheikh, ${ }^{2} \mathrm{BSc}, \mathrm{MSc}$ \\ A I Elkhalifa, ${ }^{2}$ BSc, MSc; E Elhaj, ${ }^{5} \mathrm{MD}, \mathrm{MRCP} ; \mathbf{R}$ R M Salih, ${ }^{6} \mathrm{BSc}, \mathrm{PhD} ; \mathbf{M}$ E Hamid, ${ }^{3} \mathrm{BVSc}, \mathrm{MSc}, \mathrm{PhD}$ \\ ${ }^{1}$ Department of Preventive Medicine, Faculty of Veterinary Medicine, University of Khartoum, Sudan \\ ${ }^{2}$ Department of Microbiology, Royale Care International Hospital, Khartoum, Sudan \\ ${ }^{3}$ Department of Microbiology, College of Medicine, King Khalid University, Abha, Kingdom of Saudi Arabia \\ ${ }^{4}$ Department of Microbiology, Faculty of Medical Laboratory Science, Omdurman Islamic University, Omdurman, Sudan \\ ${ }^{5}$ Department of Medicine, Royale Care International Hospital, Khartoum, Sudan \\ ${ }^{6}$ Department of Clinical Medicine, Faculty of Veterinary Medicine, University of Khartoum, Sudan
}

Corresponding author: M E Hamid (mehamid2@yahoo.com)

We report a case of pulmonary nocardiosis in a 41-year-old asthma patient. Chest radiographs showed bilateral air space and consolidations. Acid-fast branching filaments were demonstrated in sputum, and the grown organism was identified phenotypically and confirmed using 16S rDNA sequencing (accession no. KX500116). The patient received a combination of medical treatments, but developed complications, which were managed over the next 3 months, after which she was discharged. Pulmonary nocardiosis should be considered in patients undergoing steroid therapy or when a chronic infection does not respond to first-line treatment.

S Afr Med J 2017;107(1):43-45. DOI:10.7196/SAMJ.2017.v107i1.12006

Nocardiae (genus Nocardia) are aerobic, Gram-positive, mycolic acid-containing actinomycetes and characteristically form acidalcohol-fast branching filaments. They are saprophytes, but have been known to cause invasive and disseminated infections in humans and animals for $>100$ years. ${ }^{[1,2]}$ Infections caused by N. otitidiscaviarum are rare $(3 \%)$ compared with those caused by other Nocardia spp. Infection in humans was first reported in the mid-1960s and the first systemic infection in humans was reported in $1974,{ }^{[3,4]}$ when Nocardia sp. was diagnosed in the lungs of a 76-year-old man who had asthma since the age of 6 years. His asthma problem worsened owing to pulmonary nocardiosis or prolonged steroid therapy. ${ }^{[5]}$

The diagnosis of pulmonary nocardiosis based on clinical and radiological features is problematic, as these resemble other lung pathogens, including Mycobacterium tuberculosis. There is a dearth of knowledge in the epidemiological, clinical presentations, outcomes and laboratory information of nocardiosis, which challenge its importance as a threatening health risk. ${ }^{[2,6]}$ This report aimed to identify a unique isolate that caused a severe illness in a female asthmatic patient.

\section{Case report}

We report the case of a 41-year-old woman with severe pneumonia. She had an 18-month history of bronchial asthma and underwent a cholecystectomy 5 years previously. On admission, the patient was suffering from bronchial asthma and severe pneumonia, with a productive cough (yellow sputum) of $>3$ months' duration. She complained of high-grade fever and appeared ill, with dyspnoea and wheezing. Crepitations could be heard over the right lower zone of the chest. An electrocardiogram (ECG) indicated sinus tachycardia, abnormal rhythm and possible atrial enlargement.

A computed tomography (CT) scan of the chest showed bilateral asymmetrical patchy air space disease, more evident on the right side. There was consolidation on the middle lobe and bilateral pleural effusion, but no cavities. These signs are suggestive of atypical pneumonia.

Chest radiographs of the patient indicated consolidation on the right lower lobe, left lobular infiltration and right pleural effusion (Fig. 1).

Pleural aspirates were collected using ultrasound guidance. The pleural fluid appeared bloody, with a white blood cell count of $10000 / \mu \mathrm{L}$, comprising $80 \%$ neutrophils and $20 \%$ lymphocytes. The protein content was $3.09 \mathrm{~g} / \mathrm{dL}$ and glucose was $168 \mathrm{mg} / \mathrm{dL}$. A Ziehl-Neelsen-stained smear was made from the aspirate and from a sputum sample. The smear showed acid-fast branching filaments (Fig. 2) suggestive of Nocardia infection.

On admission the patient was administered cefuroxime and clarithromycin. The following day, after we received the laboratory report, the patient was administered sulfamethoxazole 3 tablets (160 mg) 3 times daily, and amikacin $500 \mathrm{mg}$ and imipenem $500 \mathrm{mg}$ intravenously. Ten days later the patient developed pancytopenia. The sulfamethoxazole and amikacin regimen was discontinued. The patient was administered ceftriaxone $2 \mathrm{~g}$ intravenously twice daily plus imipenem $500 \mathrm{mg}$ intravenously and was given a blood transfusion. On receiving the results of the laboratory antimicrobial assay, we started the patient on ceftriaxone $2 \mathrm{~g}$ intravenously twice daily, amikacin $500 \mathrm{mg}$ intravenously twice daily plus ciprofloxacin $500 \mathrm{mg}$ orally twice daily. Three days later she was progressing well, and no crepitations were detected. The patient recovered, but had a dry cough and crepitations for a while.

Growth of the causative agent was obtained from both sputum and pleural aspirate specimens on blood agar (BBL Microbiology Inc., USA) after 3 days under aerobic conditions at $30^{\circ} \mathrm{C}$. Subsequently, the organism was sub-cultured for identification on other bacterio- 


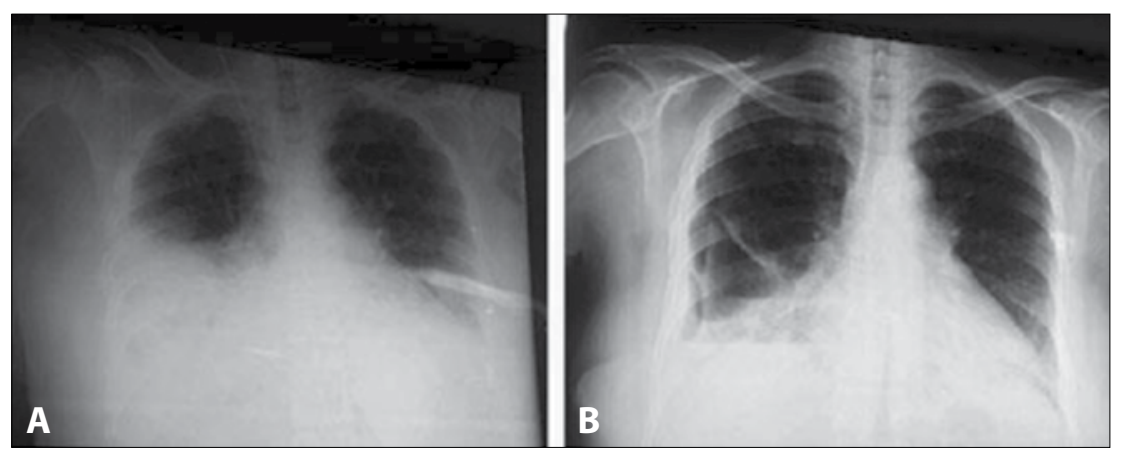

Fig. 1. Chest radiograph of the 41-year-old patient, showing right lower-lobe consolidation and left lobular infiltration. There is also right pleural effusion. (A) Anteroposterior view. (B) Posteroanterior view.

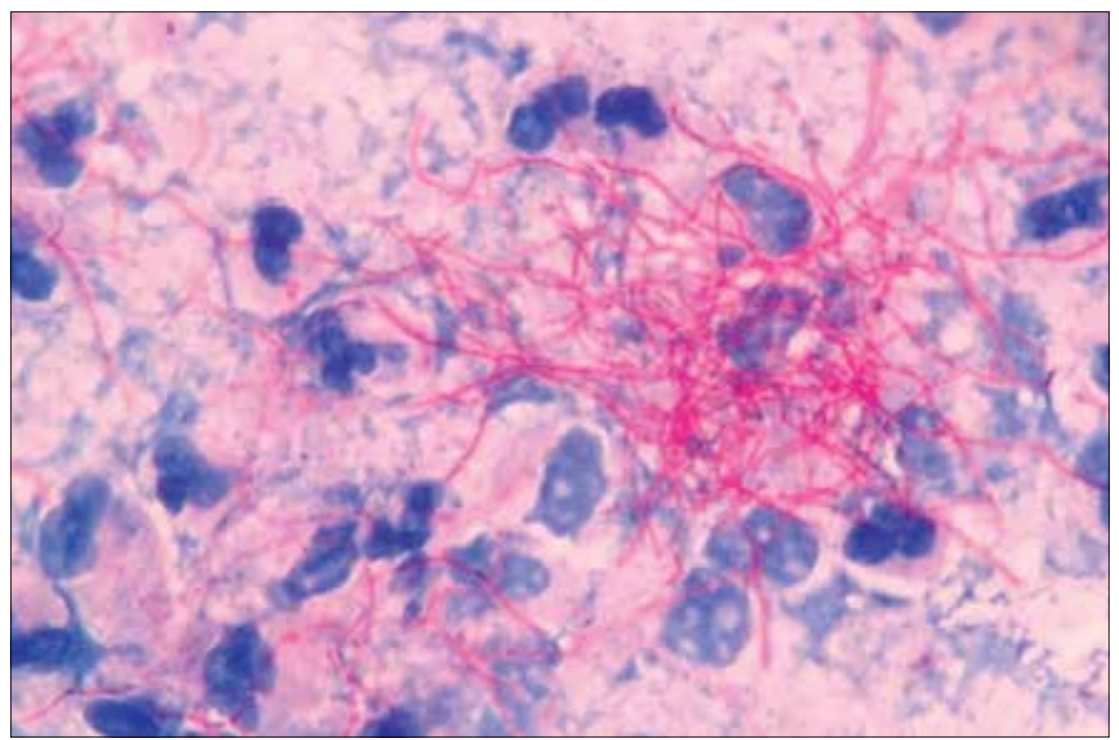

Fig. 2. A Ziehl-Neelsen-stained smear, which was obtained from sputum of the 41-year-old female patient with pulmonary infection. The smear shows partially acid-fast branching filaments, which fragmented into short chains; notice the many inflammatory and granulomatous cells.
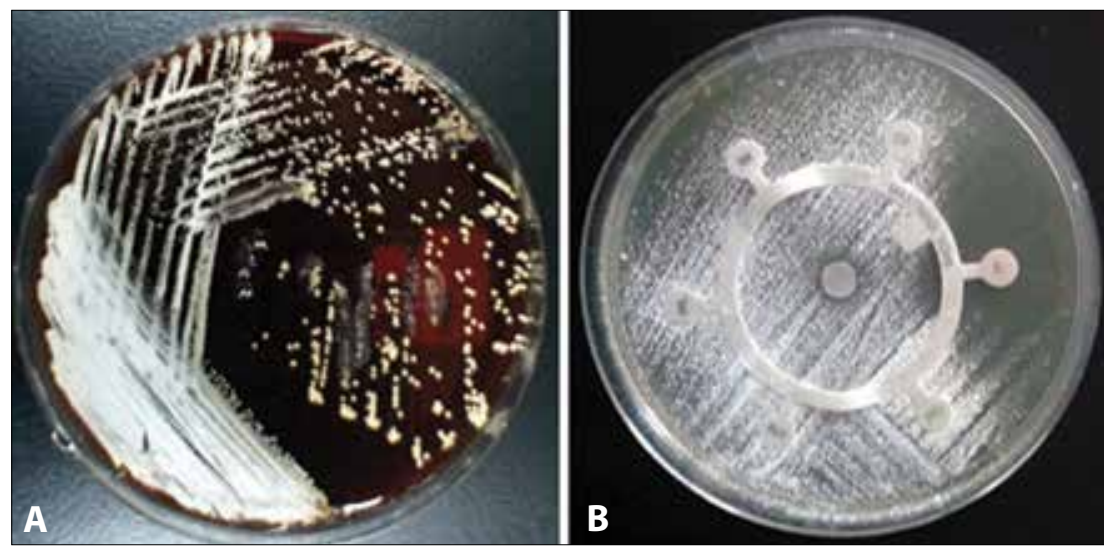

Fig. 3. Growth of isolate SD16 on blood agar after 7 days under aerobic conditions. (A) The growth indicates actinomycete-type organisms with chalky grey-white, rough, wrinkled colonies, which are embedded into agar. (B) The antimicrobial assay shows clear sensitivity of the isolate to amikacin. The dark zone of inhibition is discernable around the amikacin-impregnated disk.

logical media, including glucose malt extract, yeast extract agar, tryptic soya agar and Löwenstein-Jensen media. The morphological properties of the grown culture indicated an actinomycete-type organism with a chalky grey-white, rough, wrinkled appearance deeply embedded into agar (Fig. 3). Smears made from the culture revealed Gram-positive and partially acidfast branching filamentous organisms when stained with the modified Ziehl-Neelsen method. The filaments appeared as short chains of rods and coccobacillary elements, which are characteristic for organisms of the genus Nocardia. ${ }^{[1]}$ The organism displayed in vitro sensitivity to amikacin, cefditoren, ciprofloxacin, meropenem and streptomycin, but resistance to azithromycin, erythromycin, ceftazidime, penicillin, rifampin, and sulfamethoxazole/trimethoprim (Fig. 3).

Six days later Acinetobacter spp. were isolated from the sputum, which were found to be sensitive to only colistin, a clear multidrug-resistant nosocomial complication. The patient was then administered colistin intravenously and nebulised. She improved, sputum culture was negative, colistin was discontinued and she was discharged.

16S rDNA nucleotide sequences data (1473 bp; Genbank (National Institutes of Health, USA) accession no. KX500116) were first analysed using the basic local alignment search tool (BLAST, USA) system ${ }^{[7]}$ to determine a swift phylogenetic position. After designation of the isolate with Nocardia spp. in the BLAST system, the sequences were analysed using Molecular Evolutionary Genetics Analysis version 6.0 (MEGA6) software (Center for Evolutionary Medicine and Informatics, USA $)^{[8]}$ and compared with all known sequences of Nocardia spp. found in the GenBank database. ${ }^{[9]}$ The isolate SD16 was identical to that of N. otitidiscaviarum in the $16 \mathrm{~S}$ rDNA gene phylogenetic tree (Fig. 4).

Our patient had signed an informed consent form, indicating her agreement to participate in the study.

\section{Discussion}

Pulmonary nocardiosis is diagnosed in a considerable number of patients who suffer from chronic lung diseases in African countries. ${ }^{[10]}$ Previously, we noticed that Nocardia spp. cause pulmonary infections in both immunocompetent and immunocompromised patients. ${ }^{[11]}$ Pulmonary nocardiosis is a rare opportunistic infection that predominantly affects immunocompromised patients. A high clinical suspicion is vital for an early diagnosis and appropriate treatment in such patients, who present with chest involvement or signs of chronic pneumonia. ${ }^{[12]}$

$N$. otitidiscaviarum is commonly resistant to beta-lactams, including most broad-spectrum cephalosporins, amoxicillin-clavulanic acid, and imipenem, but are usually susceptible to amikacin, fluoroquinolones, and sulfonamides. ${ }^{[6,9]}$ The isolate showed in vitro antibiotic susceptibilities similar to those reported previously. ${ }^{[6]}$ Our patient was treated empirically with trimethoprim-sulfamethoxazole, but it was discontinued on receiving 


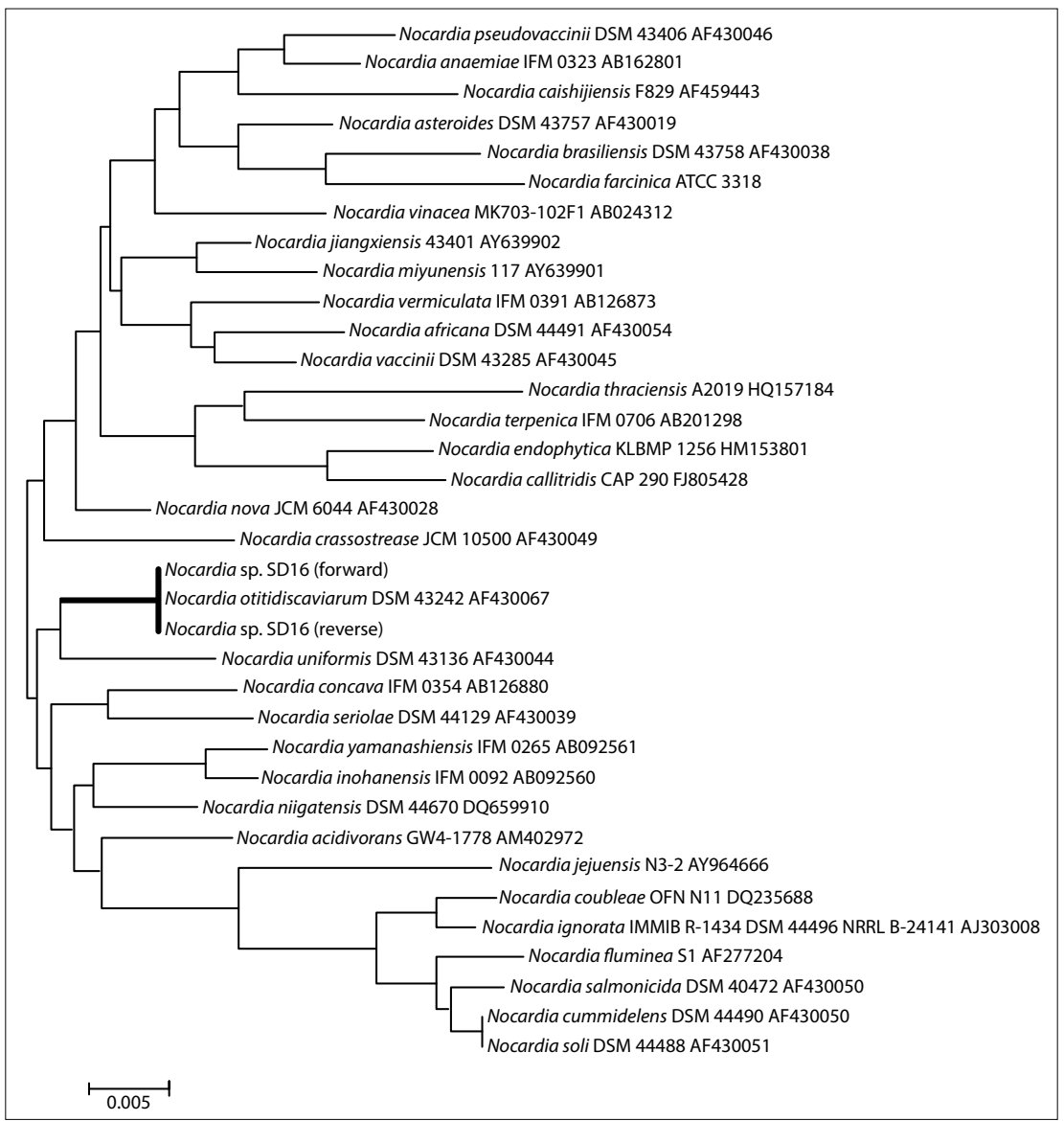

Fig. 4. A phylogenetic tree showing the relationship of Nocardia sp. strain SD16 (reverse and forward sequences) with the majority of related Nocardia spp. (bold bar). The tree was determined using the Neighbor-Joining method. All positions containing gaps and missing data were eliminated. Evolutionary analysis was performed using MEGA6 software. ${ }^{[s]}$ The scale bar indicates 0.005 substitution per nucleotide position.

the laboratory report. Complications and difficulties with regard to treatment seem similar to those in a previously reported pulmonary lowed by imipenem/cilastatin, amikacin and minocycline. ${ }^{[5]}$

Acknowledgements. We are grateful to all staff of the microbiology department at Royal Care International Hospital, Khartoum, Sudan, notably to Ms M K Sivaprakasam, Mr A A Hassan and Mr M Azhari.

1. Goodfellow M, Maldonado LA. Genus Nocardia trevisan 1889AL. In: Goodfellow M, Kampfer P, Busse HJ, et al., eds. Bergey's Manual of Systematic Bacteriology, vol. 5. 2nd ed. New York: Springer, 2012:376-419. http://dx.doi.org/10.1007/978-0387-68233-4

2. Kandi V. Human Nocardia infections: A review of pulmonary nocardiosis. Cureus 2015;7(8):e304. http://dx.doi.org/10.7759/ cureus. 304

3. Arroyo JC, Nichols S, Carroll GF. Disseminated Nocardia caviae infection. Am J Med 1977;62(3):409-412.

. Causey WA, Arnell P, Brinker J. Systemic Nocardia caviae infection. Chest 1974;65(3):360-362.

5. Bandoh C, Amano M, Suzuki M, Aoki S, Matsuoka R. A case of pulmonary nocardiosis during steroid therapy for asthma. Nihon Kokyuki Gakkai Zasshi 2008;46(12):1024-1028.

6. Amin A, Mahmood SF, Anis M, et al. Pulmonary nocardiosis: A comparative analysis of Nocardia asteroides and non-aste-
A colin A comparative analysis of Nocardia asteroides and non-aste-
roides species. Trop Doct 2012;42(2):94-96. http://dx.doi roides species. Trop Dot

7. National Center for Biotechnology Information. Basic local National Center for Biotechnology Information. Basic local
alignment search tool. 2016. http://blast.ncbi.nlm.nih.gov/Blast.cgi (accessed November 2016)

8. Tamura K, Stecher G, Peterson D, Filipski A, Kumar S. MEGA6 Molecular Evolutionary Genetics Analysis version 6.0. Mo Biol Evol 2013;30(12):2725-2729. http://dx.doi.org/10.1093/ molbev/mst197

9. National Center for Biotechnology Information. Nucleotide. 2016. https://www.ncbi.nlm.nih.gov/nucleotide/ (accessed November 2016).

10. Hamid ME, Maldonado L, Sharaf Eldin GS, Mohamed MF, Saeed NS, Goodfellow M. Nocardia africana sp. nov., a new pathogen isolated from patients with pulmonary infections. J Clin Microbiol 2001;39(2):625-630. http://dx.doi.org/10.1128//CM.39.2.625biol $2001 ; 39$

11. Alnaum HM, Elhassan MM, Mustafa FY, Hamid ME Prevalence of Nocardia species among HIV-positive patients with suspected tuberculosis. Trop Doct 2011;41(4):224-226. http://dx.doi.org/10.1258/td.2011.110107

12. Chen J, Zhou H, Xu P, Zhang P, Ma S, Zhou J. Clinical an radiographic characteristics of pulmonary nocardiosis: Clues to earlier diagnosis. PLoS ONE 2014;9(3):e90724. http://dx.doi. org/10.1097/MD.0000000000001561

nocardiosis patient with asthma, ${ }^{[5]}$ who was treated with a series of antimicrobials, including sulfamethoxazole and trimethoprim, fol- 